\title{
Linking generalized parton distributions to constituent quark models
}

\author{
S. Boffi ${ }^{a}$, B. Pasquini ${ }^{b},{ }^{c}$, M. Traini ${ }^{c}$ \\ ${ }^{a}$ Dipartimento di Fisica Nucleare e Teorica, Università degli Studi di Pavia \\ and INFN, Sezione di Pavia, Pavia, Italy \\ ${ }^{b} \mathrm{ECT}^{*}$, Villazzano (Trento), Italy \\ ${ }^{c}$ Dipartimento di Fisica, Università degli Studi di Trento, Povo (Trento), and \\ INFN, Sezione di Trento, Trento, Italy
}

\begin{abstract}
The link between the nucleon generalized parton distributions and the non-diagonal one-body density matrix in momentum space is studied. Attention is focussed on the region where quark generalized parton distributions (GPD's) describe emission and reabsorption of a single active quark by the target nucleon. The correct covariant connection with wave functions used in any constituent quark model is established. Results obtained with different constituent quark models are presented for the unpolarized quark GPD's.
\end{abstract}

Key words: generalized parton distributions, constituent quark models PACS 12.39.-x, 13.60.Fz, 13.60.Hb, 14.20.Dh

\section{Introduction}

In recent years much theoretical activity has been devoted to generalized parton distributions (GPD's). They are defined as nondiagonal hadronic matrix elements of bilocal products of the light-front quark and gluon field operators and they interpolate between the inclusive physics of parton distributions and

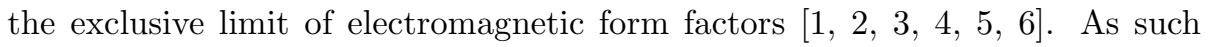
they contain information on parton correlations and the internal spin structure of the nucleon. But the main interest to study GPD's comes from the fact that, due to the factorization theorem [5, 7, 8], they are candidate to provide us with a unifying theoretical background suitable to describe a variety of inclusive and exclusive processes in the deep inelastic scattering (DIS) regime. In particular, they enter the cross section for exclusive photon production, i.e. deeply virtual Compton scattering (DVCS) [4, 5, 9, 10], and hard diffractive electroproduction of (longitudinal) vector and pseudo-scalar meson 111. In addition, in the forward limit they become diagonal matrix elements giving the usual DIS parton 
distribution, and their first moment gives the nucleon elastic form factors [曰]. Basic properties of the GPD's have been reviewed in Refs. [12, 13, 14].

Much effort is underway related to the measurement of these functions. A general discussion about the problems arising in the experimental study of the GPD's and the first experimental evidences have been presented in Ref. 115]. Prospects for future measurements of DVCS are studied in Ref. [16]. On the other hand, model calculations are a necessary prerequisite to define suitable strategies to extract GPD's from experiments. In the literature there are two approaches used to model the nucleon GPD's. One is a phenomenological construction based on reduction formulas where GPD's are related to the usual parton distributions by factorizing the momentum transfer dependence due to the nucleon electroweak form factors [17, 13, 18]. This leads to double distribution functions parametrizing the nonforward matrix elements involved in DVCS and hard exclusive electroproduction processes discussed, e.g., in Ref. [19]. Another approach is based on direct calculation of GPD's in specific dynamical models. The first model calculations were performed using the MIT bag model 20]. Further calculations have been performed in the chiral quark-soliton model [21, 22].

A complete and exact overlap representation of GPD's has been recently worked out within the framework of light-cone quantisation [23, 24]. In particular, quark GPD's are obtained as overlaps of light-cone wave functions (LCWF's) indicating that they are deeply connected with the non-diagonal one-body density matrix in momentum space occurring in any nonrelativistic many-body problem. A preliminar investigation of such an approach was presented in Ref. 25].

In fact, direct calculation of LCWF's from first principles is a difficult task. On the other hand, constituent quark models (CQM's) have been quite successful in describing the spectrum of hadrons and their low-energy dynamics. Therefore it is interesting to explore the connection between GPD's and CQM wave functions at least in the allowed kinematic range where only quark degrees of freedom are effective. This corresponds to the kinematic region where GPD's describe how a quark is taken out from the proton and, having undergone a hard scattering, is inserted back as a quark inside the scattered proton. This connection has been studied in Ref. [26] where a simple nonrelativistic quark model has been used to calculate one of the twist-two GPD's at a low-energy scale. However, as in the case of parton distributions calculated from CQM wave functions a nonrelativistic approach is obviously insufficient [27] and a consequence of it is that the obtained GPD's are not defined on their natural support and the particle number and momentum sum rules are not always preserved. The support violation occurs also with the MIT bag model 20 because the initial and final nucleons are not good momentum eigenstates. These are common problems arising also in the case of parton distributions calculated from CQM wave functions [28, 29] where they were cured by appropriately taking into account translation invariance [30].

CQM's rely on quantum theory with a finite number of degrees of freedom. In this case relativity can be incorporated quite naturally by utilizing relativistic Hamiltonian dynamics [31 and the Bakamjian-Thomas [32] construction of the 
Hamiltonian for a system of interacting particles. In such an approach CQM wave functions can be considered as eigenfunctions of the nucleon Hamiltonian in the instant-form dynamics and can simply be related to wave functions in any form of relativistic Hamiltonian dynamics. Examples are the light-front description of electromagnetic form factors (see Ref. 33] and references therein) and the covariant calculation of the nucleon electroweak form factors [34] in a chiral CQM 35 with the point-form approach. Similarly, one can obtain a link between CQM wave functions and LCWF's with the corresponding transformation from the instant-form to the front-form representation as it is here proposed. GPD's in the allowed kinematic region are then obtained in a covariant approach and they exhibit the exact forward limit reproducing the parton distribution with the correct support and automatically fulfilling the particle number and momentum sum rules.

The paper is organized as follows. In Sect. 2 the relevant definitions and properties of GPD's in terms of LCWF's are recalled. In Sect. 3 the connection is established between the (light-cone) front-form and the (canonical) instantform description. This connection is used in Sect. 4 to obtain the unpolarized GPD's and their forward limits, i.e. the usual DIS parton distributions in terms of CQM wave functions. The results obtained with different CQM wave functions are presented in Sect. 5, confining some technical details in the Appendix, and concluding remarks are given in Sect. 6 .

\section{The unpolarized generalized parton distribu- tions}

For definiteness let us consider virtual Compton scattering where a lepton exchanges a virtual photon of momentum $q^{\mu}$ with a nucleon of momentum $P^{\mu}$, producing a real photon of momentum $q^{\prime \mu}$ and a recoil nucleon of momentum $P^{\prime \mu}$. Ultimately we are here interested in kinematic conditions similar to those familiar in the DIS regime that is characterized by the Bjorken limit, i.e. $Q^{2}=-q^{2} \rightarrow \infty, P \cdot q \rightarrow \infty$, and fixed $x_{B}=Q^{2} / 2(P \cdot q)$. When focusing on the deeply virtual kinematic regime of $q^{\mu}$, i.e. DVCS, a generalization of the Bjorken kinematics is considered, namely the c.m. energy $s=(P+q)^{2}$ and the photon virtuality $Q^{2}$ are large while the invariant momentum square

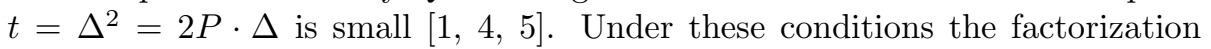
theorem [5, 7, 81 tells us that the amplitude factorizes in a hard scattering part (which is exactly calculable in perturbative QCD) and a soft, nonperturbative nucleon structure part. The contribution of the hard scattering part to leading order corresponds to the so called handbag diagram with photon scattering on a single parton (quark or antiquark). Consequently, the soft part is a quarkquark correlation function, representing the process where a parton is taken out of the initial nucleon and reinserted back into the final nucleon after hard scattering. To describe such a process, according to Ref. [4] it is useful to choose a symmetric frame of reference where the virtual photon momentum $q^{\mu}$ and 
the average nucleon momentum $\bar{P}^{\mu}=\frac{1}{2}\left(P^{\mu}+P^{\prime \mu}\right)$ are collinear along the $z$ axis and in opposite directions (Fig. 1). It is also useful to use the component notation $a^{\mu}=\left[a^{+}, a^{-}, \vec{a}_{\perp}\right]$ for any four-vector $a^{\mu}$ with light-cone components $a^{ \pm}=\left(a^{0} \pm a^{3}\right) / \sqrt{2}$ and the transverse part $\vec{a}_{\perp}=\left(a^{1}, a^{2}\right)$.

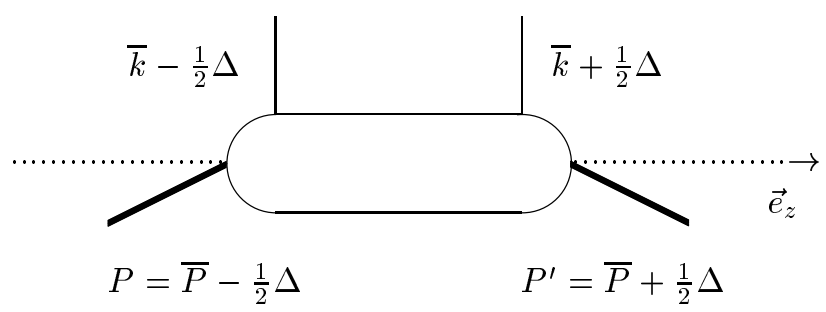

Figure 1: The symmetric frame of reference.

In the following we only consider the case of unpolarized quarks inside the nucleon. Then for each flavor $q$ the soft amplitude in Fig. 1 becomes

$$
F_{\lambda^{\prime} \lambda}^{q}(\bar{x}, \xi, t)=\left.\frac{1}{4 \pi} \int d y^{-} e^{i \bar{x} \bar{P}^{+} y^{-}}\left\langle P^{\prime}, \lambda^{\prime}\left|\bar{\psi}\left(-\frac{1}{2} y\right) \not h \psi\left(\frac{1}{2} y\right)\right| P, \lambda\right\rangle\right|_{y^{+}=\vec{y}_{\perp}=0}
$$

where $n$ is a lightlike vector proportional to $(1,0,0,-1), \lambda\left(\lambda^{\prime}\right)$ is the helicity of the initial (final) nucleon and the quark-quark correlation function is integrated along the light-cone distance $y^{-}$at equal light-cone time $\left(y^{+}=0\right)$ and at zero transverse separation $\left(\vec{y}_{\perp}=0\right)$ between the quarks. The resulting onedimensional Fourier integral along the light-cone distance $y^{-}$is with respect to the quark light-cone momentum $\bar{k}^{+}=\bar{x}^{+}$. The so called skewedness parameter $\xi$ describes the longitudinal change of the nucleon momentum, $2 \xi=-\Delta^{+} / \bar{P}^{+}$. The link operator normally needed to make the definition (1) gauge invariant does not appear because we also choose the gauge $A^{+}=0$ which reduces the link operator to unity.

Following Ref. [4] the leading twist (twist-two) part of this amplitude can be parametrized as

$$
\begin{aligned}
F_{\lambda^{\prime} \lambda}^{q}(\bar{x}, \xi, t)= & \frac{1}{2 \bar{P}^{+}} \bar{u}\left(P^{\prime}, \lambda^{\prime}\right) \gamma^{+} u(P, \lambda) H^{q}(\bar{x}, \xi, t) \\
& +\frac{1}{2 \bar{P}^{+}} \bar{u}\left(P^{\prime}, \lambda^{\prime}\right) \frac{i \sigma^{+\nu} \Delta_{\nu}}{2 M} u(P, \lambda) E^{q}(\bar{x}, \xi, t),
\end{aligned}
$$

where $u(P, \lambda)$ is the nucleon Dirac spinor and $H^{q}(\bar{x}, \xi, t)$ and $E^{q}(\bar{x}, \xi, t)$ are the chiral-even (helicity conserving) and chiral-odd (helicity flip) GPD's for partons of flavor $q$, respectively.

An explicit expression in term of LCWF's can be obtained following the lines 
of Refs. [23, 24. The hadronic state is written as

$$
|P, \lambda\rangle=\sum_{N, \beta} \int\left[\frac{d \bar{x}}{\sqrt{\bar{x}}}\right]_{N}\left[d \vec{k}_{\perp}\right]_{N} \Psi_{\lambda, N, \beta}(r)\left|N, \beta ; k_{1}, \ldots, k_{N}\right\rangle,
$$

where the integration measures are defined by

$$
\begin{gathered}
{\left[\frac{d \bar{x}}{\sqrt{\bar{x}}}\right]_{N}=\left(\prod_{i=1}^{N} \frac{d \bar{x}_{i}}{\sqrt{\bar{x}_{i}}}\right) \delta\left(1-\sum_{i=1}^{N} \bar{x}_{i}\right),} \\
{\left[d \vec{k}_{\perp}\right]_{N}=\left(\prod_{i=1}^{N} \frac{d \vec{k}_{\perp i}}{2(2 \pi)^{3}}\right) 2(2 \pi)^{3} \delta\left(\sum_{i=1}^{N} \vec{k}_{\perp i}-\vec{P}_{\perp}\right) .}
\end{gathered}
$$

The function $\Psi_{\lambda, N, \beta}(r)$ is the momentum LCWF of the $N$-parton Fock state $\left|N, \beta ; k_{1}, \ldots, k_{N}\right\rangle$ (also including gluons), with $\beta$ indicating flavour, helicity and colour quantum numbers. The LCWF's depend on the momentum coordinates (collectively indicated by $r$ ) of the partons relative to the hadron momentum. The $N$-parton states are normalized as

$$
\begin{aligned}
& \left\langle N^{\prime}, \beta^{\prime} ; k_{1}^{\prime}, \ldots, k_{N}^{\prime} \mid N, \beta ; k_{1}, \ldots, k_{N}\right\rangle \\
& \quad=\delta_{N^{\prime} N} \delta_{\beta^{\prime} \beta} \prod_{i=1}^{N} 2 k_{i}^{+}(2 \pi)^{3} \delta\left({k_{i}^{\prime}}^{+}-k_{i}^{+}\right) \delta\left(\vec{k}_{\perp i}^{\prime}-\vec{k}_{\perp i}\right) .
\end{aligned}
$$

Correspondingly, the hadron states are covariantly normalized as

$$
\left\langle P^{\prime}, \lambda^{\prime} \mid P, \lambda\right\rangle=2 P^{+}(2 \pi)^{3} \delta\left(P^{\prime+}-P^{+}\right) \delta\left(\vec{P}_{\perp}^{\prime}-\vec{P}_{\perp}\right) \delta_{\lambda^{\prime} \lambda},
$$

with

$$
\sum_{N, \beta} \int[d \bar{x}]_{N}\left[d \vec{k}_{\perp}\right]_{N}\left|\Psi_{\lambda, N, \beta}(r)\right|^{2}=1 .
$$

Having in mind the link between GPD's and CQM wave functions we have to confine our discussion to the region $\xi<\bar{x}<1$. In this region and in the symmetric frame

$$
\begin{aligned}
F_{\lambda^{\prime} \lambda}^{q}(\bar{x}, \xi, t)= & \sum_{N, \beta}(\sqrt{1-\xi})^{2-N}(\sqrt{1+\xi})^{2-N} \sum_{j=1}^{N} \delta_{s_{j} q} \\
& \times \int[d \bar{x}]_{N}\left[d \vec{k}_{\perp}\right]_{N} \delta\left(\bar{x}-\bar{x}_{j}\right) \Psi_{\lambda^{\prime}, N, \beta}^{*}\left(r^{\prime}\right) \Psi_{\lambda, N, \beta}(r) \Theta\left(\bar{x}_{j}\right),(9)
\end{aligned}
$$

where $s_{j}$ labels the quantum numbers of the $j$ th parton, $\beta$ specifies all other quantum necessary for the $N$-parton state, and the set of kinematic variables $r, r^{\prime}$ are defined as follows: for the final struck quark,

$$
y_{j}^{\prime}=\frac{\bar{k}_{j}^{+}+\frac{1}{2} \Delta^{+}}{\bar{P}^{+}+\frac{1}{2} \Delta^{+}}=\frac{\bar{x}_{j}-\xi}{1-\xi}, \quad \vec{\kappa}_{\perp j}^{\prime}=\vec{k}_{\perp j}+\frac{1}{2} \frac{1-\bar{x}_{j}}{1-\xi} \vec{\Delta}_{\perp},
$$


for the final $N-1$ spectators $(i \neq j)$,

$$
y_{i}^{\prime}=\frac{\bar{x}_{i}}{1-\xi}, \quad \vec{\kappa}_{\perp i}^{\prime}=\vec{k}_{\perp i}-\frac{1}{2} \frac{\bar{x}_{i}}{1-\xi} \vec{\Delta}_{\perp},
$$

and for the initial struck quark

$$
y_{j}=\frac{\bar{k}_{j}^{+}-\frac{1}{2} \Delta^{+}}{\bar{P}^{+}-\frac{1}{2} \Delta^{+}}=\frac{\bar{x}_{j}+\xi}{1+\xi}, \quad \vec{\kappa}_{\perp j}=\vec{k}_{\perp j}-\frac{1}{2} \frac{1-\bar{x}_{j}}{1+\xi} \vec{\Delta}_{\perp},
$$

for the initial $N-1$ spectators $(i \neq j)$,

$$
y_{i}=\frac{\bar{x}_{i}}{1+\xi}, \vec{\kappa}_{\perp i}=\vec{k}_{\perp i}+\frac{1}{2} \frac{\bar{x}_{i}}{1+\xi} \vec{\Delta}_{\perp} .
$$

Working out the spinor products we have

$$
\begin{aligned}
F_{++}^{q}(\bar{x}, \xi, t) & =F_{--}^{q}(\bar{x}, \xi, t) \\
& =\sqrt{1-\xi^{2}} H^{q}(\bar{x}, \xi, t)-\frac{\xi^{2}}{\sqrt{1-\xi^{2}}} E^{q}(\bar{x}, \xi, t), \\
F_{-+}^{q}(\bar{x}, \xi, t) & =-\left(F_{+-}^{q}(\bar{x}, \xi, t)\right)^{*}=\eta \frac{\sqrt{t_{0}-t}}{2 M} E^{q}(\bar{x}, \xi, t),
\end{aligned}
$$

where

$$
\eta=\frac{\Delta^{1}+i \Delta^{2}}{\left|\vec{\Delta}_{\perp}\right|}
$$

and

$$
-t_{0}=\frac{4 \xi^{2} M^{2}}{1-\xi^{2}}
$$

is the minimal value for $-t$ at given $\xi$.

Therefore, one can extract $H^{q}$ and $E^{q}$ separately from the knowledge of $F_{\lambda^{\prime} \lambda}^{q}$. In particular, $E^{q}$ is directly given by Eq. (15), and

$$
H^{q}(\bar{x}, \xi, t)=\frac{1}{\sqrt{1-\xi^{2}}}\left[F_{++}^{q}(\bar{x}, \xi, t)+\frac{2 M \xi^{2}}{\eta \sqrt{t_{0}-t} \sqrt{1-\xi^{2}}} F_{-+}^{q}(\bar{x}, \xi, t)\right] .
$$

\section{Nucleon wave functions in front and instant form}

In this section a connection will be established between the (light-cone) frontform and the (canonical) instant-form description.

When necessary, labels $[f]$ and $[c]$ on the wave function will refer to front form and canonical form, respectively, and vectors with tilde are defined in the front form. 


\subsection{Single-parton states}

Omitting colour degrees of freedom that do not matter in the following considerations, single-parton states can be defined either with front-form coordinates,

$$
|\overrightarrow{\tilde{k}}, \lambda, \tau\rangle_{[f]}, \quad \overrightarrow{\vec{k}}=\left(k^{+}, \vec{k}_{\perp}\right),
$$

(with isospin $\tau$ ) or with instant-form coordinates,

$$
|\vec{k}, \lambda, \tau\rangle_{[c]}, \quad \vec{k}=\left(k_{x}, k_{y}, k_{z}\right)
$$

with corresponding normalizations

$$
\begin{aligned}
{ }_{[f]}\left\langle\overrightarrow{\tilde{k}}^{\prime}, \lambda^{\prime}, \tau^{\prime} \mid \overrightarrow{\tilde{k}}, \lambda, \tau\right\rangle_{[f]} & =2 k^{+}(2 \pi)^{3} \delta\left(k^{+}-k^{\prime+}\right) \delta\left(\vec{k}_{\perp}-\vec{k}_{\perp}^{\prime}\right) \delta_{\lambda \lambda^{\prime}} \delta_{\tau \tau^{\prime}} \\
{ }_{[c]}\left\langle\vec{k}^{\prime}, \lambda^{\prime}, \tau^{\prime} \mid \vec{k}, \lambda, \tau\right\rangle_{[c]} & =\delta\left(\vec{k}-\vec{k}^{\prime}\right) \delta_{\lambda \lambda^{\prime}} \delta_{\tau \tau^{\prime}} .
\end{aligned}
$$

The transformation from the instant- to the front-form representation reads

$$
|\overrightarrow{\tilde{k}}, \lambda, \tau\rangle_{[f]}=\sum_{\lambda^{\prime} \tau^{\prime}} \int d \vec{k}^{\prime}\left|\vec{k}^{\prime}, \lambda^{\prime}, \tau^{\prime}\right\rangle_{[c][c]}\left\langle\vec{k}^{\prime}, \lambda^{\prime}, \tau^{\prime} \mid \overrightarrow{\tilde{k}}, \lambda, \tau\right\rangle_{[f]},
$$

where, according to Eq. (4.35) of Ref. 31,

$$
{ }_{[c]}\left\langle\vec{k}^{\prime}, \lambda^{\prime}, \tau^{\prime} \mid \overrightarrow{\tilde{k}}, \lambda, \tau\right\rangle_{[f]}=\sqrt{2 \omega}(2 \pi)^{3 / 2} \delta\left(\vec{k}-\vec{k}^{\prime}\right) D_{\lambda^{\prime} \lambda}^{1 / 2}\left(R_{c f}(\overrightarrow{\tilde{k}})\right) \delta_{\tau \tau^{\prime}}
$$

Here $R_{c f}$ is a Melosh rotation, and the normalization constant $\sqrt{2 \omega}(2 \pi)^{3 / 2}$ with $\omega \equiv k_{0}=\left(k^{+}+k^{-}\right) / \sqrt{2}$ derives from a different normalization used in Ref. [31].

Then Eq. (22) becomes

$$
|\overrightarrow{\tilde{k}}, \lambda, \tau\rangle_{[f]}=\sqrt{2 \omega}(2 \pi)^{3 / 2} \sum_{\lambda^{\prime}} D_{\lambda^{\prime} \lambda}^{1 / 2}\left(R_{c f}(\overrightarrow{\tilde{k}})\right)\left|\vec{k}, \lambda^{\prime}, \tau\right\rangle_{[c]} .
$$

\subsection{N-parton states in the front form}

In the front-form description the $N$-parton contribution $|N ; \overrightarrow{\widetilde{P}}, \lambda\rangle_{[f]}$ to the nucleon wave function can be derived from the zero-momentum state by a suitable boost $L_{f}(Q)$ with $Q=|\vec{P}| / M_{0}\left(M_{0}\right.$ being the mass of the noninteracting $N$ parton system) and

$$
L_{f}(Q)_{\nu}^{\mu}(1,0,0,0)^{\nu}=\frac{P^{\mu}}{M_{0}} .
$$

In fact, under a Lorentz transformation $\Lambda$ we have

$$
U(\Lambda)|N ; \overrightarrow{\tilde{P}}, \lambda\rangle_{[f]}=\sum_{\lambda^{\prime}}\left|N ; \Lambda \overrightarrow{\tilde{P}}, \lambda^{\prime}\right\rangle_{[f]}\left\langle\lambda^{\prime}\left|R_{W}(\Lambda, P)\right| \lambda\right\rangle,
$$


where $R_{W}(\Lambda, P)$ is the Wigner rotation associated with the $\Lambda$ transformation. Assuming for $\Lambda$ the boost $\Lambda=L_{f}^{-1}(Q)$, the Wigner rotation becomes the identity, $R_{W}\left(L_{f}^{-1}(Q), P\right)=\overrightarrow{1}$. Thus

$$
\begin{gathered}
U\left(L_{f}(Q)\right)|N ; \overrightarrow{\tilde{0}}, \lambda\rangle_{[f]}=|N ; \overrightarrow{\tilde{P}}, \lambda\rangle_{[f]}, \\
U\left(L_{f}^{-1}(Q)\right)|N ; \overrightarrow{\tilde{P}}, \lambda\rangle_{[f]}=|N ; \overrightarrow{\tilde{0}}, \lambda\rangle_{[f]} .
\end{gathered}
$$

In turn, according to Eq. (24),

$$
|N ; \overrightarrow{\tilde{0}}, \lambda\rangle_{[f]}=\sqrt{2 M_{0}}(2 \pi)^{3 / 2}|N ; \overrightarrow{0}, \lambda\rangle_{[c]} .
$$

On the other hand, according to Eq. (3) we have

$$
|N ; \overrightarrow{\tilde{0}}, \lambda\rangle_{[f]}=\sum_{\tau_{i}, \lambda_{i}} \int\left[\frac{d \bar{x}}{\sqrt{\bar{x}}}\right]_{N}\left[d \vec{k}_{\perp}\right]_{N} \Psi_{\lambda}^{[f]}\left(\left\{\bar{x}_{i}, \vec{k}_{\perp i} ; \lambda_{i}, \tau_{i}\right\}\right) \prod_{i=1}^{N}\left|\overrightarrow{\tilde{k}}_{i}, \lambda_{i}, \tau_{i}\right\rangle_{[f]}
$$

\subsection{Connection with the instant-form representation}

In order to establish the connection with the instant-form representation one

has to work out the delta functions and the measures (Eqs. (44) and (5)). Since $\sum_{i} \omega_{i}=M_{0}$, we have

$$
\begin{gathered}
\delta\left(1-\sum_{i=1}^{N} \bar{x}_{i}\right)=\delta\left(1-\sum_{i=1}^{N} \frac{k_{z i}+\omega_{i}}{M_{0}}\right)=M_{0} \delta\left(\sum_{i=1}^{N} k_{z i}\right), \\
\delta\left(1-\sum_{i=1}^{N} \bar{x}_{i}\right) \delta\left(\sum_{i=1}^{N} \vec{k}_{\perp i}\right)=M_{0} \delta\left(\sum_{i=1}^{N} \vec{k}_{i}\right) .
\end{gathered}
$$

In addition,

$$
\prod_{i=1}^{N} d \bar{x}_{i} d \vec{k}_{\perp i}=\prod_{i=1}^{N} d \vec{k}_{i}\left|\frac{\partial\left(\bar{x}_{i}, \vec{k}_{\perp i}\right)}{\partial\left(\vec{k}_{i}\right)}\right|=\prod_{i=1}^{N} d \vec{k}_{i} \frac{\partial \bar{x}_{i}}{\partial k_{z i}}=\prod_{i=1}^{N} d \vec{k}_{i} \frac{\bar{x}_{i}}{\omega_{i}}
$$

We can then rewrite the zero-momentum state (30) as

$$
\begin{aligned}
|N ; \overrightarrow{\tilde{0}}, \lambda\rangle_{[f]}=\sum_{\tau_{i}, \lambda_{i}} & \int\left[\prod_{i=1}^{N} d \vec{k}_{i} \frac{\bar{x}_{i}}{\omega_{i}} \frac{1}{\sqrt{\bar{x}_{i}}} \frac{1}{2(2 \pi)^{3}}\right] 2(2 \pi)^{3} M_{0} \delta\left(\sum_{i=1}^{N} \vec{k}_{i}\right) \\
& \times \Psi_{\lambda}^{[f]}\left(\left\{\bar{x}_{i}, \vec{k}_{\perp i} ; \lambda_{i}, \tau_{i}\right\}\right) \prod_{i=1}^{N} \sqrt{2 \omega_{i}}(2 \pi)^{3 / 2} \\
& \times \sum_{\mu_{i}} D_{\mu_{i} \lambda_{i}}^{1 / 2}\left(R_{c f}\left(\overrightarrow{\tilde{k}}_{i}\right)\right)\left|\vec{k}_{i}, \mu_{i}, \tau_{i}\right\rangle_{[c]}
\end{aligned}
$$




$$
\begin{array}{r}
=\sum_{\tau_{i}, \lambda_{i}} \int\left[\prod_{i=1}^{N} d \vec{k}_{i}\left(\frac{\bar{x}_{i}}{\omega_{i}}\right)^{1 / 2}\right] M_{0} \delta\left(\sum_{i=1}^{N} \vec{k}_{i}\right) \Psi_{\lambda}^{[f]}\left(\left\{\bar{x}_{i}, \vec{k}_{\perp i} ; \lambda_{i}, \tau_{i}\right\}\right) \\
\times 2(2 \pi)^{3} \prod_{i=1}^{N}\left[2(2 \pi)^{3}\right]^{-1 / 2} \sum_{\mu_{i}} D_{\mu_{i} \lambda_{i}}^{1 / 2}\left(R_{c f}\left(\overrightarrow{\tilde{k}}_{i}\right)\right)\left|\vec{k}_{i}, \mu_{i}, \tau_{i}\right\rangle_{[c]} .(34)
\end{array}
$$

Therefore, the zero-momentum state in the canonical representation,

$$
|N ; \overrightarrow{0}, \lambda\rangle_{[c]}=\sum_{\tau_{i}, \lambda_{i}} \int \prod_{i=1}^{N} d \vec{k}_{i} \delta\left(\sum_{i=1}^{N} \vec{k}_{i}\right) \Psi_{\lambda}^{[c]}\left(\left\{\vec{k}_{i} ; \lambda_{i}, \tau_{i}\right\}\right) \prod_{i=1}^{N}\left|\vec{k}_{i}, \lambda_{i}, \tau_{i}\right\rangle_{[c]},
$$

can be rewritten in the light-front representation using the definition (29) and the result (34) as

$$
\begin{aligned}
|N ; \overrightarrow{0}, \lambda\rangle_{[c]}= & \sum_{\tau_{i}, \lambda_{i}} \int\left[\prod_{i=1}^{N} d \vec{k}_{i}\left(\frac{\bar{x}_{i}}{\omega_{i}}\right)^{1 / 2}\right] \sqrt{M_{0}} \delta\left(\sum_{i=1}^{N} \vec{k}_{i}\right) \Psi_{\lambda}^{[f]}\left(\left\{\bar{x}_{i}, \vec{k}_{\perp i} ; \lambda_{i}, \tau_{i}\right\}\right) \\
& \times\left[2(2 \pi)^{3}\right]^{1 / 2} \prod_{i=1}^{N}\left[2(2 \pi)^{3}\right]^{-1 / 2} \sum_{\mu_{i}} D_{\mu_{i} \lambda_{i}}^{1 / 2}\left(R_{c f}\left(\overrightarrow{\tilde{k}}_{i}\right)\right)\left|\vec{k}_{i}, \mu_{i}, \tau_{i}\right\rangle_{[c]} \cdot(36)
\end{aligned}
$$

From Eqs. (35) and (36) a relationship can be obtained between hadron wave functions $\Psi_{\lambda}^{[c]}$ and $\Psi_{\lambda}^{[f]}$. This relation is here worked out in the case of a nucleon with only three valence quarks, i.e. $N=3$. The three-quark (valence) Fock state of the nucleon has been shown in Ref. [36] to have only one independent LCWF for all configurations where the quark helicities add up to the helicity of the nucleon. This state is also known to almost exhaust the contribution to GPD's at large values of $\bar{x}$ [25].

By definition we have

$$
\begin{aligned}
& \Psi_{\lambda}^{[c]}\left(\vec{k}_{1}, \vec{k}_{2}, \vec{k}_{3} ; \mu_{1}, \tau_{1}, \mu_{2}, \tau_{2}, \mu_{3}, \tau_{3}\right) \\
& \quad=\left\langle\vec{k}_{1}, \mu_{1}, \tau_{1} ; \vec{k}_{2}, \mu_{2}, \tau_{2} ; \vec{k}_{3}, \mu_{3}, \tau_{3} \mid N ; \overrightarrow{0}, \lambda\right\rangle_{[c]} .
\end{aligned}
$$

The bracket on the r.h.s. can be expressed in terms of the light-front representation (36), so that

$$
\begin{aligned}
& \Psi_{\lambda}^{[c]}\left(\vec{k}_{1}, \vec{k}_{2}, \vec{k}_{3} ; \mu_{1}, \tau_{1}, \mu_{2}, \tau_{2}, \mu_{3}, \tau_{3}\right)=\frac{1}{2(2 \pi)^{3}}\left[M_{0} \frac{\bar{x}_{1} \bar{x}_{2} \bar{x}_{3}}{\omega_{1} \omega_{2} \omega_{3}}\right]^{1 / 2} \\
& \quad \times \sum_{\lambda_{1} \lambda_{2} \lambda_{3}} D_{\mu_{1} \lambda_{1}}^{1 / 2}\left(R_{c f}\left(\overrightarrow{\tilde{k}}_{1}\right)\right) D_{\mu_{2} \lambda_{2}}^{1 / 2}\left(R_{c f}\left(\overrightarrow{\tilde{k}}_{2}\right)\right) D_{\mu_{3} \lambda_{3}}^{1 / 2}\left(R_{c f}\left(\overrightarrow{\tilde{k}}_{3}\right)\right) \\
& \quad \times \Psi_{\lambda}^{[f]}\left(\bar{x}_{1}, \vec{k}_{\perp 1}, \bar{x}_{2}, \vec{k}_{\perp 2}, \bar{x}_{3}, \vec{k}_{\perp 3} ; \lambda_{1}, \tau_{1}, \lambda_{2}, \tau_{2}, \lambda_{3}, \tau_{3}\right) .
\end{aligned}
$$


Viceversa,

$$
\begin{aligned}
& \Psi_{\lambda}^{[f]}\left(\bar{x}_{1}, \vec{k}_{\perp 1}, \bar{x}_{2}, \vec{k}_{\perp 2}, \bar{x}_{3}, \vec{k}_{\perp 3} ; \lambda_{1}, \tau_{1}, \lambda_{2}, \tau_{2}, \lambda_{3}, \tau_{3}\right) \\
& =2(2 \pi)^{3}\left[\frac{1}{M_{0}} \frac{\omega_{1} \omega_{2} \omega_{3}}{\bar{x}_{1} \bar{x}_{2} \bar{x}_{3}}\right]^{1 / 2} \sum_{\mu_{1} \mu_{2} \mu_{3}} D_{\mu_{1} \lambda_{1}}^{1 / 2 *}\left(R_{c f}\left(\overrightarrow{\tilde{k}}_{1}\right)\right) D_{\mu_{2} \lambda_{2}}^{1 / 2 *}\left(R_{c f}\left(\overrightarrow{\tilde{k}}_{2}\right)\right) \\
& \quad \times D_{\mu_{3} \lambda_{3}}^{1 / 2 *}\left(R_{c f}\left(\overrightarrow{\tilde{k}}_{3}\right)\right) \Psi_{\lambda}^{[c]}\left(\vec{k}_{1}, \vec{k}_{2}, \vec{k}_{3} ; \mu_{1}, \tau_{1}, \mu_{2}, \tau_{2}, \mu_{3}, \tau_{3}\right) .
\end{aligned}
$$

Eqs. (38) and (39) give the correct covariant transformation linking wave functions obtained in the (canonical) instant form, e.g. in some CQM, and the corresponding valence-quark component of the LCWF.

\section{The valence-quark contribution}

The valence-quark contribution to GPD's is obtained by specializing Eq. (9) to the case $N=3$, i.e.

$$
\begin{aligned}
F_{\lambda^{\prime} \lambda}^{q}(\bar{x}, \xi, t)= & \frac{1}{\sqrt{1-\xi^{2}}} \sum_{\lambda_{i} \tau_{i}} \sum_{j=1}^{3} \delta_{s_{j} q} \int[d \bar{x}]_{3}\left[d \vec{k}_{\perp}\right]_{3} \delta\left(\bar{x}-\bar{x}_{j}\right) \\
& \times \Psi_{\lambda^{\prime}}^{[f] *}\left(r^{\prime},\left\{\lambda_{i}\right\},\left\{\tau_{i}\right\}\right) \Psi_{\lambda}^{[f]}\left(r,\left\{\lambda_{i}\right\},\left\{\tau_{i}\right\}\right) \Theta\left(\bar{x}_{j}\right),
\end{aligned}
$$

where the front-form wave function $\Psi_{\lambda}^{[f]}\left(r,\left\{\lambda_{i}\right\},\left\{\tau_{i}\right\}\right)$ is related to the corresponding wave function $\Psi_{\lambda}^{[c]}\left(\left\{\vec{k}_{i}\right\},\left\{\lambda_{i}\right\},\left\{\tau_{i}\right\}\right)$ in the canonical form by Eq. (39). Separating the spin-isospin from the space part of the canonical wave function,

$$
\Psi_{\lambda}^{[c]}\left(\left\{\vec{k}_{i}\right\},\left\{\lambda_{i}\right\},\left\{\tau_{i}\right\}\right)=\psi\left(\vec{k}_{1}, \vec{k}_{2}, \vec{k}_{3}\right) \Phi_{\lambda \tau}\left(\lambda_{1}, \lambda_{2}, \lambda_{3}, \tau_{1}, \tau_{2}, \tau_{3}\right),
$$

we have

$$
\begin{aligned}
\Psi_{\lambda}^{[f]}\left(r,\left\{\lambda_{i}\right\},\left\{\tau_{i}\right\}\right)=2(2 \pi)^{3}\left[\frac{1}{M_{0}} \frac{\omega_{1} \omega_{2} \omega_{3}}{\bar{x}_{1} \bar{x}_{2} \bar{x}_{3}}\right]^{1 / 2} \psi\left(\vec{k}_{1}, \vec{k}_{2}, \vec{k}_{3}\right) \\
\quad \times \sum_{\mu_{1} \mu_{2} \mu_{3}} D_{\mu_{1} \lambda_{1}}^{1 / 2 *}\left(R_{c f}\left(\vec{k}_{1}\right)\right) D_{\mu_{2} \lambda_{2}}^{1 / 2 *}\left(R_{c f}\left(\vec{k}_{2}\right)\right) D_{\mu_{3} \lambda_{3}}^{1 / 2 *}\left(R_{c f}\left(\vec{k}_{3}\right)\right) \\
\quad \times \Phi_{\lambda \tau}\left(\mu_{1}, \mu_{2}, \mu_{3}, \tau_{1}, \tau_{2}, \tau_{3}\right)
\end{aligned}
$$

where the Melosh rotations are given by

$$
\begin{aligned}
D_{\lambda \mu}^{1 / 2}\left(R_{c f}(\overrightarrow{\tilde{k}})\right) & =\left\langle\lambda\left|R_{c f}\left(\bar{x} M_{0}, \vec{k}_{\perp}\right)\right| \mu\right\rangle \\
& =\left\langle\lambda\left|\frac{m+\bar{x} M_{0}-i \vec{\sigma} \cdot\left(\hat{\vec{z}} \times \vec{k}_{\perp}\right)}{\sqrt{\left(m+\bar{x} M_{0}\right)^{2}+\vec{k}_{\perp}^{2}}}\right| \mu\right\rangle .
\end{aligned}
$$




\subsection{Parton distributions}

In the limit $\Delta^{\mu} \rightarrow 0$, where $\bar{x}$ goes over to the parton momentum fraction $x$, some of the GPD's reduce to the ordinary DIS parton distributions. In particular,

$$
H^{q}(x, 0,0)=q(x),
$$

where $q(x)$ is the (unpolarized) quark distribution of flavor $q$. In this limit, the Melosh rotation matrices combine to the identity matrix and the following simple expression is obtained for the parton distribution

$$
q(x)=\sum_{j=1}^{3} \delta_{\tau_{j} \tau_{q}} \int \prod_{i=1}^{3} d \vec{k}_{i} \delta\left(\sum_{i=1}^{3} \vec{k}_{i}\right) \delta\left(x-\frac{k_{j}^{+}}{M_{0}}\right)\left|\Psi_{\lambda}^{[c]}\left(\left\{\vec{k}_{i} ; \lambda_{i}, \tau_{i}\right\}\right)\right|^{2} .
$$

This expression agrees with that given, e.g., in Refs. [37, 38] and automatically fulfills the support condition, vanishing outside the support region $0 \leq x \leq 1$. It also satisfies the particle number sum rule,

$$
\int d x q(x)=N_{q}
$$

where $N_{q}$ is the number of valence quarks of flavor $q$, as well as the momentum sum rule

$$
\int d x x[u(x)+d(x)]=1,
$$

where $u(x)$ and $d(x)$ are the up and down quark distributions.

\section{Results and discussion}

In this Section we present results obtained with two constituent quark models, i.e. the relativistic hypercentral quark model of Ref. [27] and the Goldstoneboson-exchange (GBE) model of Ref. [35].

\subsection{Adopted models}

The hypercentral model is based on the mass operator $M=M_{0}+V$, where $M_{0}$ is the free mass operator,

$$
M_{0}=\sum_{i=1}^{3} \sqrt{\vec{k}_{i}^{2}+m_{i}^{2}}
$$

with $\sum_{i} \vec{k}_{i}=0$, and $m_{i}$ being the constituent quark masses. The interaction $V$ is taken of the form 39

$$
V=-\frac{\tau}{y}+\kappa_{l} y
$$


where $y=\sqrt{\vec{\rho}^{2}+\vec{\lambda}^{2}}$ is the radius of the hypersphere in six dimensions and $\vec{\rho}$ and $\vec{\lambda}$ are the Jacobi coordinates,

$$
\vec{\rho}=\frac{\vec{r}_{1}-\vec{r}_{2}}{\sqrt{2}}, \quad \vec{\lambda}=\frac{\vec{r}_{1}+\vec{r}_{2}-2 \vec{r}_{3}}{\sqrt{6}} .
$$

The model depends on two parameters, $\tau$ and $\kappa_{l}$, and is able to reproduce the basic features of the low-lying nucleon spectrum satisfactorily in spite of its simplicity. In the hypercentral model the resulting nucleon wave function is a product of a space and a spin-isospin part and is $\mathrm{SU}(6)$ symmetric. Technical details concerning the derivation of the relevant formulae with this model are given in the Appendix.

In the GBE model the same semirelativistic free mass operator $M_{0}$ of Eq. (48) is combined with an interaction between constituent quarks which is the sum of a (linear) color-electric confinement term and a hyperfine (flavor-dependent) potential provided by the possible exchange of all mesons of the pseudoscalar octet and singlet. Such mesons are considered as Goldstone bosons appearing in the model as a consequence of the spontaneous breaking of chiral symmetry. The model of Ref. [35] depends on five parameters that are fixed by looking at the baryon spectrum which is well reproduced up to $2 \mathrm{GeV}$ with the correct orderings of the positive- and negative-parity states in the light and strange sectors. The resulting nucleon wave functions, without any further parameter, yield a remarkably consistent picture of the electroweak form factors [34].

\subsection{Results}

The spin-averaged $\left(H^{q}\right)$ and the helicity-flip $\left(E^{q}\right)$ GPD's calculated in the GBE model for the $u$ and $d$ flavours are plotted in Figs. 20 as a function of $\bar{x}$ at different values of $t$ and $\xi$. They are all positive apart from $E^{d}$ that is always negative. This is in agreement with the findings within the MIT bag model [20] and also within the chiral quark-soliton model [21, 22] where $H^{u}+H^{d}$ and $E^{u}-E^{d}$ are leading order in the number of colours $N_{c}$, while $H^{u}-H^{d}$ and $E^{u}+E^{d}$ are subleading. In all cases the GPD's vanish at $\bar{x}=\xi$ since in our approach they include the contribution of valence quarks only $23,25$. . They also vanish beyond $\bar{x}=1$ satisfying the support condition as already mentioned. As in the MIT bag model [20] the $\xi$ dependence at fixed $t$, Figs. 3 and 4 , turns out to be weak, the main effect being a small shift towards larger $\bar{x}$ with increasing $\xi$. In addition an interesting feature emerges from Fig. 2: the large $t$-independence of both $E^{q}$ and $H^{q}$ at fixed $\xi$ in the region $x>0.5$. Such a result is not consistent with the $t$ dependence simply factorized in terms of nucleon form factors [18]. Actually, only the first moments of the GPD's are related to the nucleon elastic form factors 伍, i.e.

$$
\int_{-1}^{1} d x H^{q}(x, \xi, t)=F_{1}^{q}(t), \quad \int_{-1}^{1} d x E^{q}(x, \xi, t)=F_{2}^{q}(t),
$$


where $F_{1}^{q}(t)$ and $F_{2}^{q}(t)$ are the contribution of quark $q$ to the Dirac and Pauli form factors. This property holds in the present approach, for $\xi=0$ only. The $\xi$ dependence which emerges from Figs. 2 1 1 in calculating the first moment of $E^{q}$ and $H^{q}$ is due to the fact that only the valence-quark contribution is taken into account. The inclusion of higher order configurations in the Fock space would restore the validity of Eq. (51) for $\xi>0$.

It is worthwhile noticing that neglecting the effect of the Melosh rotations in the transformation from the canonical- to the front-form wave function, Eq. (42), the helicity-flip GPD's $E^{q}$ would be vanishing for wave functions with $\mathrm{S}$ waves only. The use of the correct transformation can also be appreciated when looking at the parton distributions for $u$ and $d$ flavours plotted in Fig. 5 . Here the fully covariant light-front calculation gives contrasting results with those obtained with the prescription of Ref. [30] including the support and flux factor corrections. To preserve normalization the quite substantial shift of the peak to lower $\bar{x}$ values of the light-front calculation is compensated by a higher tail at larger $\bar{x}$ which reflects the presence of high-momentum components in the GBE wave functions.

GPD's calculated with the hypercentral and the GBE model behave quite similarly in spite of the fact that the hypercentral model is $\mathrm{SU}(6)$ symmetric, while the GBE model is not. However, it is known that the $\mathrm{SU}(6)$ breaking part in the nucleon GBE wave function is small 34, 35. In Fig. 6 the GPD's obtained within the hypercentral model are shown for $\xi=0$ as a function of $t$. In order to better appreciate the difference between the GBE and hypercentral CQM's, Fig. 7 shows the $t$ dependence of the ratio between $E^{q}$ and the anomalous magnetic moments $k^{q}$ predicted by the respective models $\left(k^{q}=\int d x E^{q}(x, t=0, \xi=0)\right)$. The numerical values for $k^{q}$ are listed in the caption of Fig. 7 and discussed in the text later on.

In Fig. 8 the GPD's obtained within the hypercentral model are shown for $t=-0.5(\mathrm{GeV})^{2}$ as a function of $\xi$. They confirm the results obtained within the GBE model. At large $\bar{x}$, where it is known that the contribution of valence quarks is substantial and higher Fock states are less important 25], the distributions are almost independent of $\xi$ so that at fixed $t$ their peak position for increasing $\xi$ is shifted to higher values of $\bar{x}$ by the requirement that they have to vanish here at $\bar{x}=\xi$. Would higher Fock states (including gluons and antiquarks) be included, nonvanishing GPD's should occur for $\bar{x}<\xi$ with $\bar{x}=\xi$ as a cross-over point (see, e.g., Ref. [21, 22]).

Finally we discuss the integral properties of $H^{q}$ and $E^{q}$ summarized in Eq. (51). In Fig. 9 the proton and neutron Pauli and Dirac form factors are shown as function of $-t$ in both the hypercentral and GBE models. The $F_{2}$ form factor at $t=0$ gives the following values for the nucleon anomalous magnetic moments: $k_{G B E}^{p}=1.20, k_{G B E}^{n}=-1.06, k_{\text {hyp }}^{p}=0.91, k_{\text {hyp }}^{n}=-0.82$. These results are rather far from the experimental values and consistent with analogous light-front calculations when pointlike structure of the quark is assumed [33, 40, 41]. The $\mathrm{SU}(6)$ breaking effects which produce deviations from the value $k^{p} / k^{n}=-1$ come mainly from the Melosh rotations, with a small additional contribution from the quark wave functions in the case of the GBE 
model. The $t$ dependence of $F_{1}$ and $F_{2}$ is rather smooth and in both models it does not reproduce the experimental results: a clear limitation due to the assumed pointlike structure of the quarks which can be solved by the introduction of specific quark form factors [33, 41].

\section{Concluding remarks}

A fully covariant approach has been presented linking the overlap representation of generalized parton distributions [23, 24, to the non-diagonal one-body density matrix in momentum space. As a result of the correct transformation of the wave functions from the (canonical) instant-form to the (light-cone) front-form description, the support condition is automatically fulfilled and the particle number and momentum sum rules are also satisfied. The method has been applied to the case of nucleon wave functions involving only the three valence quarks in order to study GPD's in terms of constituent quark model wave functions. This implies that the discussion of GPD's has to be confined to the region $\xi \leq \bar{x} \leq 1$. In this region one can easily derive the usual parton distributions and appreciate the advantages of a fully covariant treatment avoiding problems connected with the support condition and the flux factor introduced in other approaches. In addition, the helicity flip GPD's naturally arise thanks to the relativistic effects of the Melosh rotations. However, the limitation $\xi \leq \bar{x} \leq 1$ prevents the possibility of testing the reduction formula of the first moment of the GPD's leading to the nucleon form factors for $\xi \neq 0$.

Results have been presented for the hypercentral model of Ref. 27] and the Goldstone-boson-exchange model of Ref. [35]. Quite similar results are obtained within the two models, the $\mathrm{SU}(6)$ breaking contribution present in the GBE wave functions being rather small. A strong $t$ and a weak $\xi$ dependence has been found in all cases, confirming the results obtained within the MIT bag model [20]. Keeping in mind the limitation $\xi \leq \bar{x} \leq 1$ due to the inclusion of the lowest order Fock-space components with three valence quarks only, the results obtained show that all the phenomenology for large $\bar{x}$ and small $t$ can be studied within the present approach.

\section{Acknowledgements}

We acknowledge M. Diehl for useful comments.

\section{A Appendix}

The structure of the nucleon wave function in the hypercentral quark model of Ref. 27 is given by Eq. (41) where a product is assumed of a symmetric function of the momenta with a symmetric function of the spin-isospin variables as in Eq. (41). The spin function $\Phi_{\lambda}^{S_{12}}\left(\lambda_{1}, \lambda_{2}, \lambda_{3}\right)$ is symmetric or antisymmetric under the interchange of quark 1 and quark 2 for the total spin of the pair 
$S_{12}=1$ and $S_{12}=0$, respectively. The third quark spin is then coupled to $S_{12}$ to obtain the nucleon spin $\frac{1}{2}$. The same is done for isospin according to $\mathrm{SU}(2)$ symmetry. The resulting spin-isospin function,

$$
\begin{aligned}
& \Phi_{\lambda \tau}\left(\lambda_{1}, \lambda_{2}, \lambda_{3}, \tau_{1}, \tau_{2}, \tau_{3}\right) \\
& \quad=\frac{1}{\sqrt{2}}\left[\Phi_{\lambda}^{0}\left(\lambda_{1}, \lambda_{2}, \lambda_{3}\right) \Phi_{\tau}^{0}\left(\tau_{1}, \tau_{2}, \tau_{3}\right)+\Phi_{\lambda}^{1}\left(\lambda_{1}, \lambda_{2}, \lambda_{3}\right) \Phi_{\tau}^{1}\left(\tau_{1}, \tau_{2}, \tau_{3}\right)\right]
\end{aligned}
$$

is then fully symmetric under all permutations. The space part of the nucleon wave function is taken with total orbital momentum $L=0$ and is written in momentum representation as

$$
\psi\left(\vec{k}_{1}, \vec{k}_{2}, \vec{k}_{3}\right)=\psi_{00}(\tilde{y}) Y_{[0,0,0]}^{(0,0)}(\Omega),
$$

where $\psi_{\gamma, \nu}(\tilde{y})$ is the hyperradial wave function solution in momentum space of an eigenvalue problem for the mass operator $M$ as a sum of the free mass operator (48) and the hypercentral potential given by Eq. (49). The hyperspherical harmonics $Y_{\left[\gamma, l_{\rho}, l_{\lambda}\right]}^{(L, M)}$ are defined on the hypersphere of unit radius.

Such wave function is transformed to a front-form wave function according to Eq. (42) and inserted into Eq. (40). The summation over isospin variables gives $\delta_{T_{12} 0} \delta_{\tau_{3} 1 / 2}+\delta_{T_{12} 1}\left[\delta_{\tau_{3} 1 / 2}+2 \delta_{\tau_{3}-1 / 2}\right] / 3$ for the proton and $\delta_{T_{12} 0} \delta_{\tau_{3}-1 / 2}+$ $\delta_{T_{12} 1}\left[2 \delta_{\tau_{3} 1 / 2}+\delta_{\tau_{3}-1 / 2}\right] / 3$ for the neutron.

Summation over spin variables requires much more effort because of the presence of the Melosh rotations but is straightforward along the lines of Ref. 442. For the proton we can finally rewrite Eq. (40) as

$$
\begin{aligned}
F_{\lambda^{\prime} \lambda}^{q}=\frac{3}{2} & \frac{1}{\sqrt{1-\xi^{2}}} \frac{1}{\left(16 \pi^{3}\right)^{2}} \int \prod_{1=1}^{3} d \bar{x}_{i} \delta\left(1-\sum_{i=1}^{3} \bar{x}_{i}\right) \delta\left(\bar{x}-\bar{x}_{3}\right) \\
& \times \int \prod_{i=1}^{3} d^{2} \vec{k}_{\perp, i} \delta\left(\sum_{i=1}^{3} \vec{k}_{\perp, i}\right) \tilde{\psi}^{*}\left(\left\{y_{i}^{\prime}\right\},\left\{\vec{\kappa}_{\perp, i}^{\prime}\right\}\right) \tilde{\psi}\left(\left\{y_{i}\right\},\left\{\vec{\kappa}_{\perp, i}\right\}\right) \\
& \times \delta_{\tau_{q} \tau_{3}}\left\{X_{\lambda^{\prime} \lambda}^{00}\left(\vec{\kappa}^{\prime}, \overrightarrow{\tilde{\kappa}}\right) \delta_{\tau_{3} 1 / 2}+\frac{1}{3} X_{\lambda^{\prime} \lambda}^{11}\left(\vec{\kappa}^{\prime}, \overrightarrow{\tilde{\kappa}}\right)\left[\delta_{\tau_{3} 1 / 2}+2 \delta_{\tau_{3}-1 / 2}\right]\right\}(54)
\end{aligned}
$$

where

$$
\begin{gathered}
\tilde{\psi}\left(\left\{x_{i}\right\},\left\{\vec{k}_{\perp, i}\right\}\right)=\left[\frac{1}{M_{0}} \frac{\omega_{1} \omega_{2} \omega_{3}}{x_{1} x_{2} x_{3}}\right] \psi\left(\vec{k}_{1}, \vec{k}_{2}, \vec{k}_{3}\right), \\
X_{\lambda^{\prime} \lambda}^{00}\left(\overrightarrow{\tilde{k}}^{\prime}, \overrightarrow{\tilde{k}}\right)=\prod_{i=1}^{3} N^{-1}\left(\overrightarrow{\tilde{k}}_{i}^{\prime}\right) N^{-1}\left(\overrightarrow{\tilde{k}}_{i}\right)\left\langle\lambda^{\prime}\left|A_{3}+i \vec{B}_{3} \cdot \vec{\sigma}\right| \lambda\right\rangle\left(A_{1} A_{2}+\vec{B}_{1} \cdot \vec{B}_{2}\right), \\
X_{\lambda^{\prime} \lambda}^{11}\left(\overrightarrow{\tilde{k}}^{\prime}, \overrightarrow{\tilde{k}}\right)=\prod_{i=1}^{3} N^{-1}\left(\overrightarrow{\tilde{k}}_{i}^{\prime}\right) N^{-1}\left(\overrightarrow{\tilde{k}}_{i}\right) \frac{1}{3} \sum_{j j^{\prime}}\left[\left(A_{1} A_{2}-\vec{B}_{1} \cdot \vec{B}_{2}\right) \delta_{j j^{\prime}}\right.
\end{gathered}
$$




$$
\begin{aligned}
+ & \left.B_{1, j} B_{2, j^{\prime}}+B_{1, j^{\prime}} B_{2, j}+\sum_{k}\left(A_{1} B_{2, k}+A_{2} B_{1, k}\right) \epsilon_{j^{\prime} j k}\right] \\
\times & \left\langle\lambda^{\prime}\right| A_{3} \delta_{j j^{\prime}}+\sum_{k} \epsilon_{j^{\prime} j k} B_{3, k}+i\left(\sum_{k} A_{3} \epsilon_{j^{\prime} j k} \sigma_{k}\right. \\
& \left.+\sigma_{j^{\prime}} B_{3, j}+\sigma_{j} B_{3, j^{\prime}}-\vec{\sigma} \cdot \vec{B}_{3} \delta_{j j^{\prime}}\right)|\lambda\rangle
\end{aligned}
$$

with

$$
\begin{gathered}
N(\overrightarrow{\tilde{k}})=\left[\left(m+x M_{0}\right)^{2}+\vec{k}_{\perp}^{2}\right]^{1 / 2}, \\
A_{i}=\left(m+x_{i}^{\prime} M_{0}^{\prime}\right)\left(m+x_{i} M_{0}\right)+k_{y}^{\prime} k_{y}+k_{x}^{\prime} k_{x}, \\
B_{i, x}=-\left(m+x_{i}^{\prime} M_{0}^{\prime}\right) k_{y}+\left(m+x_{i} M_{0}\right) k_{y}^{\prime}, \\
B_{i, y}=\left(m+x_{i}^{\prime} M_{0}^{\prime}\right) k_{x}-\left(m+x_{i} M_{0}\right) k_{x}^{\prime}, \\
B_{i, z}=k_{x}^{\prime} k_{y}-k_{y}^{\prime} k_{x} .
\end{gathered}
$$

In the above equations the primed (unprimed) kinematical variables refer to the final (initial) nucleon state (see Eqs. (10) and (12)) and:

$$
M_{0}=\sum_{i} \sqrt{\vec{k}_{i}^{2}+m_{i}^{2}}, \quad M_{0}^{\prime}=\sum_{i} \sqrt{\vec{k}_{i}^{\prime 2}+m_{i}^{2}} .
$$

In the GBE model the nucleon wave functions are expanded on a basis where the spin-isospin part is combined with a space part in the form of correlated gaussian functions of the Jacobi coordinates referring to a particular partition. The total wave function is a symmetrized linear combination of such basis functions over the three possible partitions thus ultimately violating $\mathrm{SU}(6)$ symmetry. Specifically, we have

$$
\Psi_{\lambda}^{[c]}\left(\left\{\vec{k}_{i}\right\},\left\{\lambda_{i}\right\},\left\{\tau_{i}\right\}\right)=\sum_{n} c_{n} \Psi_{\alpha_{n}}\left(\left\{\vec{k}_{i}\right\},\left\{\lambda_{i}\right\},\left\{\tau_{i}\right\}\right),
$$

where each $\Psi_{\alpha}$ is summed over all possible partitions, i.e.

$$
\Psi_{\alpha}=\Psi_{\alpha}(1,23)+\Psi_{\alpha}(2,31)+\Psi_{\alpha}(3,12) .
$$

In turn, for each partition $(k, p q)$

$$
\Psi_{\alpha}(k, p q)=\left[\psi_{L M}\left(\vec{x}_{k}, \vec{y}_{k}\right) \bigotimes \Phi_{\lambda}\left(\lambda_{p}, \lambda_{q}, \lambda_{k}\right)\right]_{J} \Phi_{\tau}\left(\tau_{p}, \tau_{q}, \tau_{k}\right),
$$

where

$$
\psi_{L M}\left(\vec{x}_{k}, \vec{y}_{k}\right)=x_{k}^{2 \nu+\lambda} y_{k}^{2 n+l} e^{-\beta x_{k}^{2}-\delta y_{k}^{2}+\gamma \vec{x}_{k} \cdot \vec{y}_{k}} Y_{\lambda l}^{L M}\left(\hat{x}_{k}, \hat{y}_{k}\right),
$$

with $2 \nu+\lambda+2 n+l=2 N+L, N$ being the principal quantum number, and

$$
Y_{\lambda l}^{L M}\left(\hat{x}_{k}, \hat{y}_{k}\right)=\sum_{\mu m}(\lambda l \mu m \mid L M) Y_{\lambda \mu}\left(\hat{x}_{k}\right) Y_{l m}\left(\hat{y}_{k}\right) .
$$


In the above equations $\vec{x}_{k}, \vec{y}_{k}$ are the Jacobi coordinates of partition $k$. The parameters $\beta, \delta$ and $\gamma$ are fixed by solving the eigenvalue problem according to, e.g., the stochastic variational method [43] as in Ref. [35]. For the nucleon, $N=L=0$.

The calculation with the GBE wave function requires repeating the same steps as with the hypercentral wave functions for each partition of the partial contribution to the total initial (final) nucleon wave function (64).

\section{References}

[1] D. Müller, D. Robaschik, B. Geyer, F.M. Dittes, J. Hořejs̆i, Fortsch. Phys. 42 (1994) 101.

[2] A.V. Radyushkin, Phys. Lett. B 380 (1996) 417.

[3] A.V. Radyushkin, Phys. Lett. B 385 (1996) 333.

[4] Xiangdong Ji, Phys. Rev. Lett. 78 (1997) 610.

[5] A.V. Radyushkin, Phys. Rev. D 56 (1997) 5524.

[6] M. Diehl, Eur. Phys. J. C 19 (2001) 485.

[7] X. Ji and J. Osborne, Phys. Rev. D 58 (1998) 094018.

[8] J.C. Collins and A. Freund, Phys. Rev. D 59 (1999) 074009.

[9] Xiangdong Ji, Phys. Rev. D 55 (1997) 7114.

[10] A.V. Belitsky, D. Müller and A. Kirchner, Nucl. Phys. B 629 (2002) 323.

[11] J.C. Collins, L.L. Frankfurt and M. Strikman, D 56 (1997) 2982.

[12] Xiangdong Ji, J. Phys. G 24 (1998) 1181.

[13] A.V. Radyushkin, hep-ph/0101225.

[14] K. Goeke, M.V. Polyakov and M. Vanderhaeghen, Progr. Part. Nucl. Phys. 47 (2001) 401.

[15] M. Guidal, Nucl. Phys. A699 (2002) 200.

[16] V.A. Korotkov and W.-D. Nowak, Eur. Phys. J. C 23 (2002) 455.

[17] A.V. Radyushkin, Phys. Rev. D 59 (1999) 014030.

[18] M.Vanderhaeghen, P.A.M. Guichon and M. Guidal, Phys. Rev. D 60 (1999) 094017.

[19] M. Vanderhaeghen, Eur. Phys. J. A 8 (2000) 455.

[20] X. Ji, W. Melnitchouk and X. Song, Phys. Rev. D 56 (1997) 5511. 
[21] V.Yu. Petrov, P.V. Pobylitsa, M.V. Polyakov, I. Börnig, K. Goeke and C. Weiss, Phys. Rev. D 57 (1998) 4325.

[22] M. Penttinen, M.V. Polyakov and K. Goeke, Phys. Rev. D 62 (2000) 014024.

[23] M. Diehl, Th. Feldmann, R. Jakob and P. Kroll, Nucl. Phys. B 596 (2001) 33.

[24] S.J. Brodsky, M. Diehl and D.S. Hwang, Nucl. Phys. B 596 (2001) 99.

[25] M. Diehl Th. Feldmann, P. Kroll and R. Jakob, Eur. Phys. J. C 8 (1999) 409.

[26] S. Scopetta and V. Vento, hep-ph/0201265.

[27] P. Faccioli, M. Traini and V. Vento, Nucl. Phys. A 656 (1999) 400.

[28] L. Conci and M. Traini, Few-Body Systems, 8, 123 (1990); M. Traini, L. Conci and U. Moschella, Nucl. Phys. A 544 (1992) 731.

[29] F.M. Steffens and A.W. Thomas, Nucl. Phys. A 568 (1994) 798.

[30] M. Traini, V. Vento, A. Mair and A. Zambarda, Nucl. Phys. A 614 (1997) 472; A. Mair and M. Traini, Nucl. Phys. A 624 (1997) 564.

[31] B.D. Keister and W.N. Polyzou, Adv. Nucl. Phys. 20 (1991) 225.

[32] B. Bakamjian and L.H. Thomas, Phys. Rev. 92 (1953) 1300.

[33] F. Cardarelli, E. Pace, G. Salmè, and S. Simula, Phys. Lett B 357 (1995) 267.

[34] R.F. Wagenbrunn, S. Boffi, W. Klink, W. Plessas and M. Radici, Phys. Lett. B 511 (2001) 33; L.Ya. Glozman, M. Radici, R.F. Wagenbrunn, S. Boffi and W. Plessas, Phys. Lett. B 516 (2001) 183; S. Boffi, L.Ya. Glozman, W. Klink, W. Plessas, M. Radici and R.F. Wagenbrunn, Eur. Phys. J. A 14 (2002) 17-21.

[35] L.Ya. Glozman, W. Plessas, K. Varga and R.F. Wagenbrunn, Phys. Rev. D 58 (1998) 094030.

[36] Z. Dziembowski, Phys. Rev. D 37 (1988) 768.

[37] S.J. Brodsky, H.-Ch. Pauli and S.S. Pinsky, Phys. Rep. 301 (1998) 299.

[38] S.J. Brodsky and G.P. Lepage, in: A.H. Mueller (ed.), Perturbative Quantum Chromodynamics, World Scientific, Singapore, 1989, p.93.

[39] The hypercentral-potential model has been introduced, within a nonrelativistic framework, in: M. Ferraris, M.M. Giannini, M. Pizzo, E. Santopinto, L. Tiator, Phys. Lett. B 364 (1995) 231. 
[40] B. Pasquini, M. Traini and S. Boffi, Phys. Rev. D 65 (2002) 074028.

[41] S. Simula, in: D. Drechsel and L. Tiator (eds.), NSTAR 2001, Proceedings of the Workshop on the Physics of Excited Nucleons, World Scientific, Singapore, 2001, p.135, and nucl-th/0105024

[42] P.L. Chung and F. Coester, Phys. Rev. D 44 (1991) 229.

[43] Y. Suzuki and K. Varga, Stochastic Variational Approach to QuantumMechanical Few-Body Problems, (Springer Verlag, Berlin/Heidelberg, 1998). 


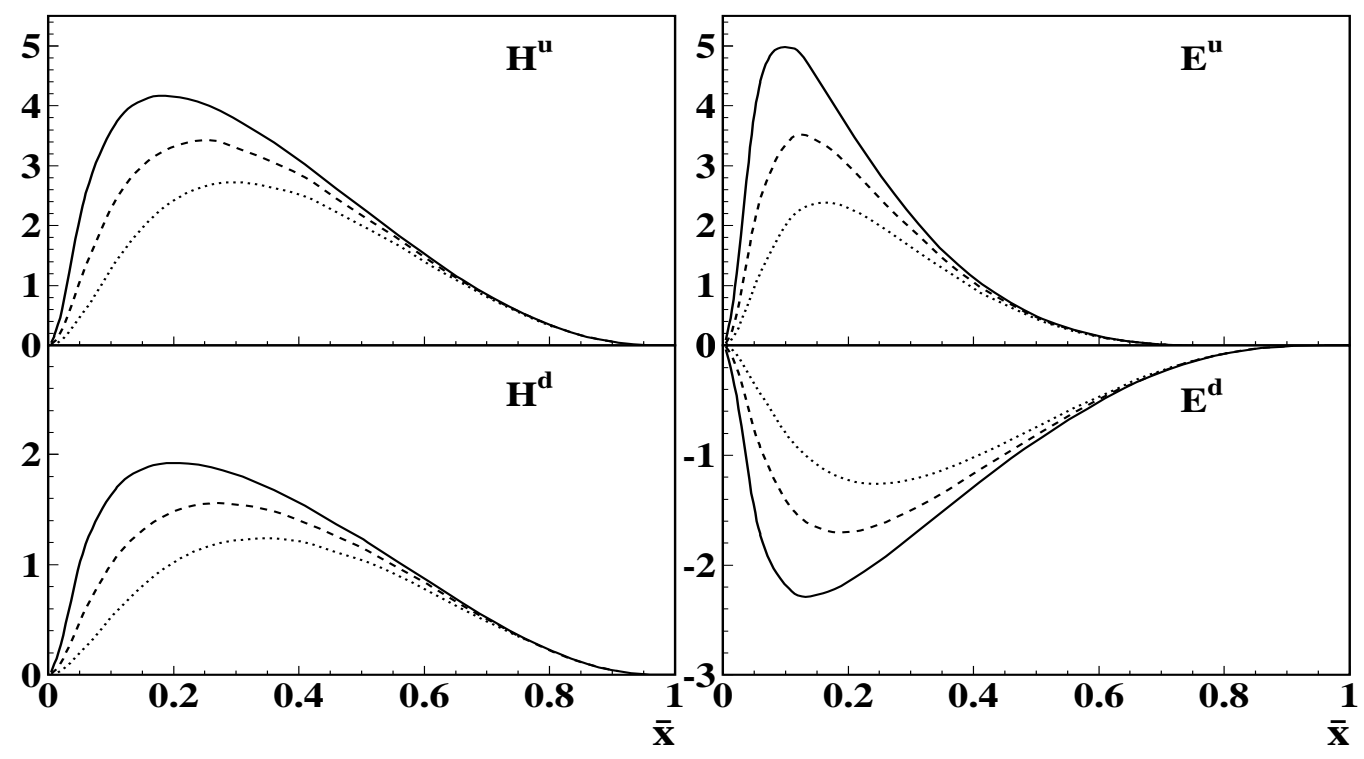

Figure 2: The spin-averaged ( $H^{q}$, left panels) and the helicity-flip ( $E^{q}$, right panels) generalized parton distributions calculated in the GBE model for flavours $u$ (upper panels) and $d$ (lower panels), at $\xi=0$ and different values of $t: t=0$ (solid curves), $t=-0.2(\mathrm{GeV})^{2}$ (dashed curves), $t=-0.5(\mathrm{GeV})^{2}$ (dotted curves). 

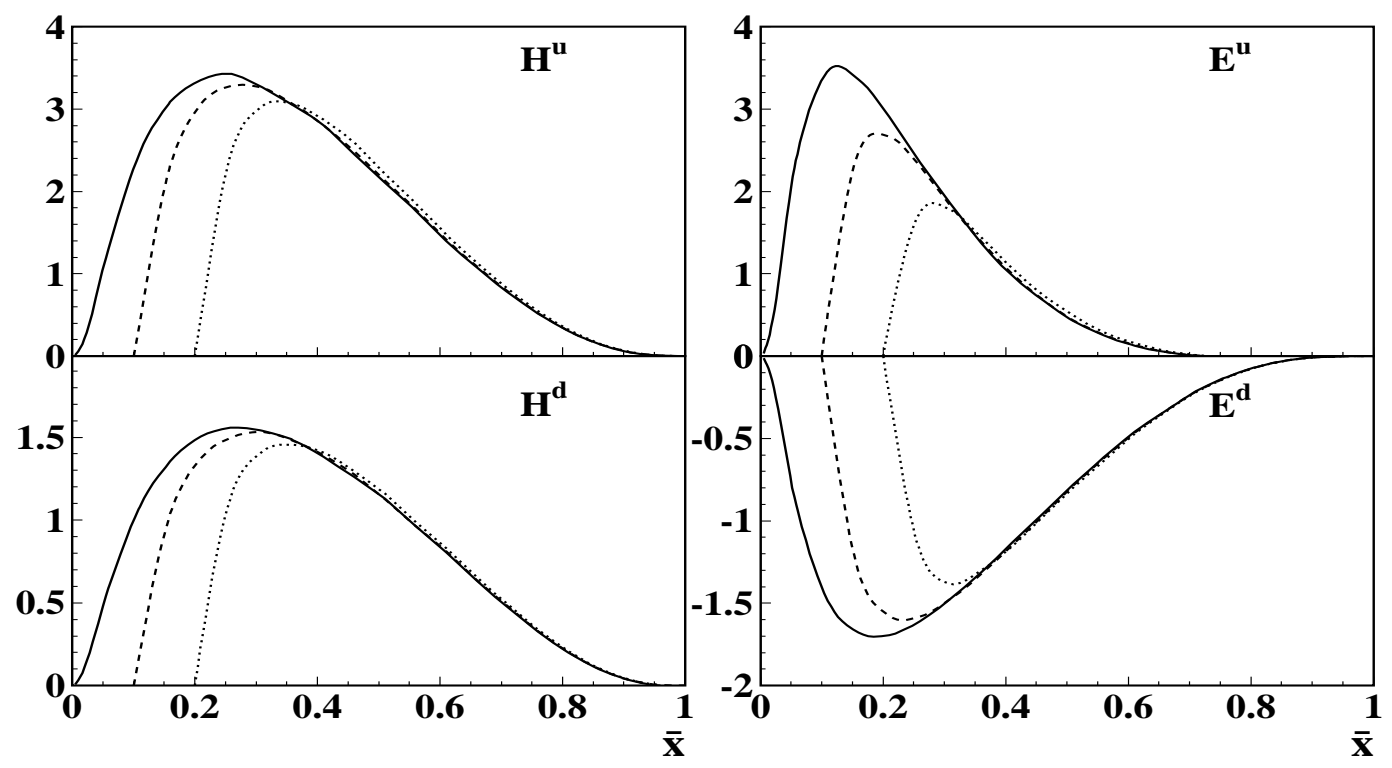

Figure 3: The same as in Fig. 2, but for fixed $t=-0.2(\mathrm{GeV})^{2}$ and different values of $\xi: \xi=0$ (solid curves), $\xi=0.1$ (dashed curves), $\xi=0.2$ (dotted curves). 


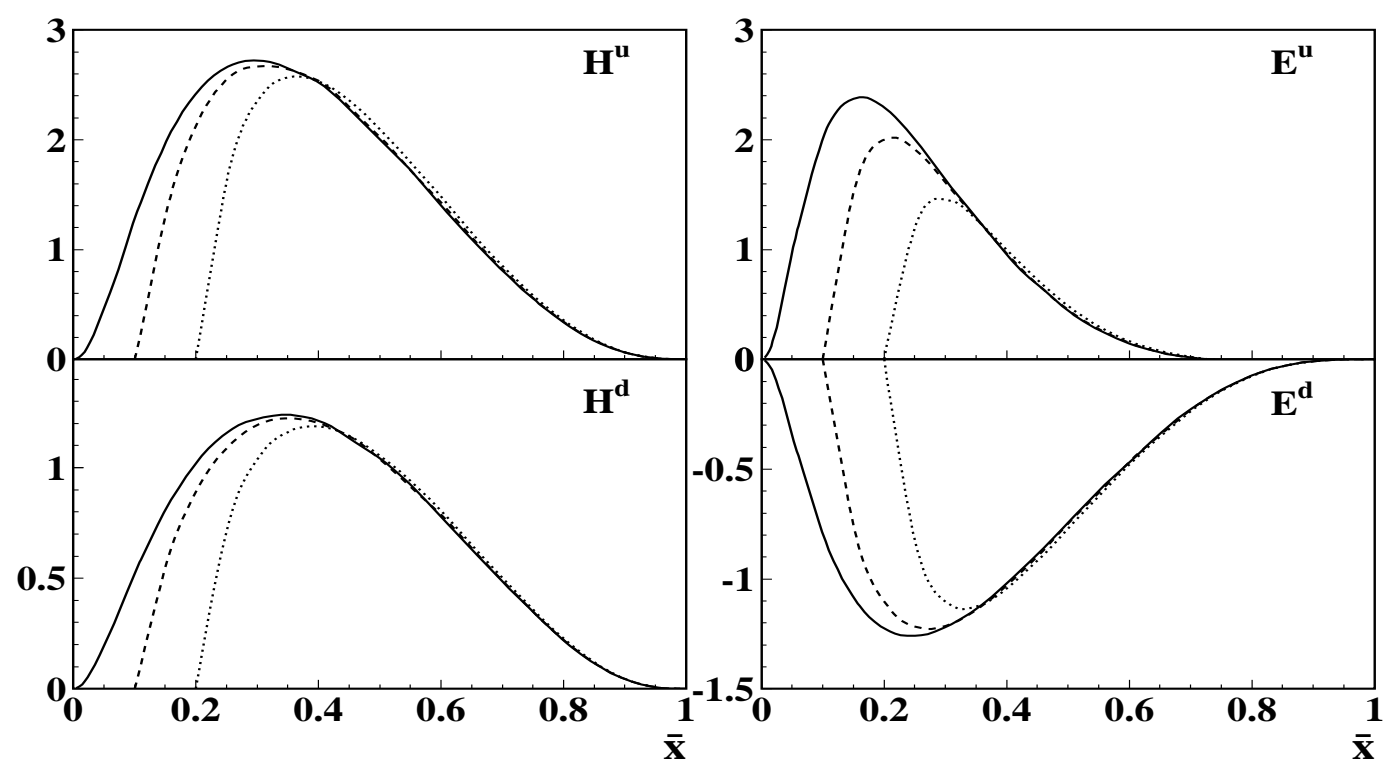

Figure 4: The same as in Fig. 2, but for fixed $t=-0.5(\mathrm{GeV})^{2}$ and different values of $\xi: \xi=0$ (solid curves), $\xi=0.1$ (dashed curves), $\xi=0.2$ (dotted curves).
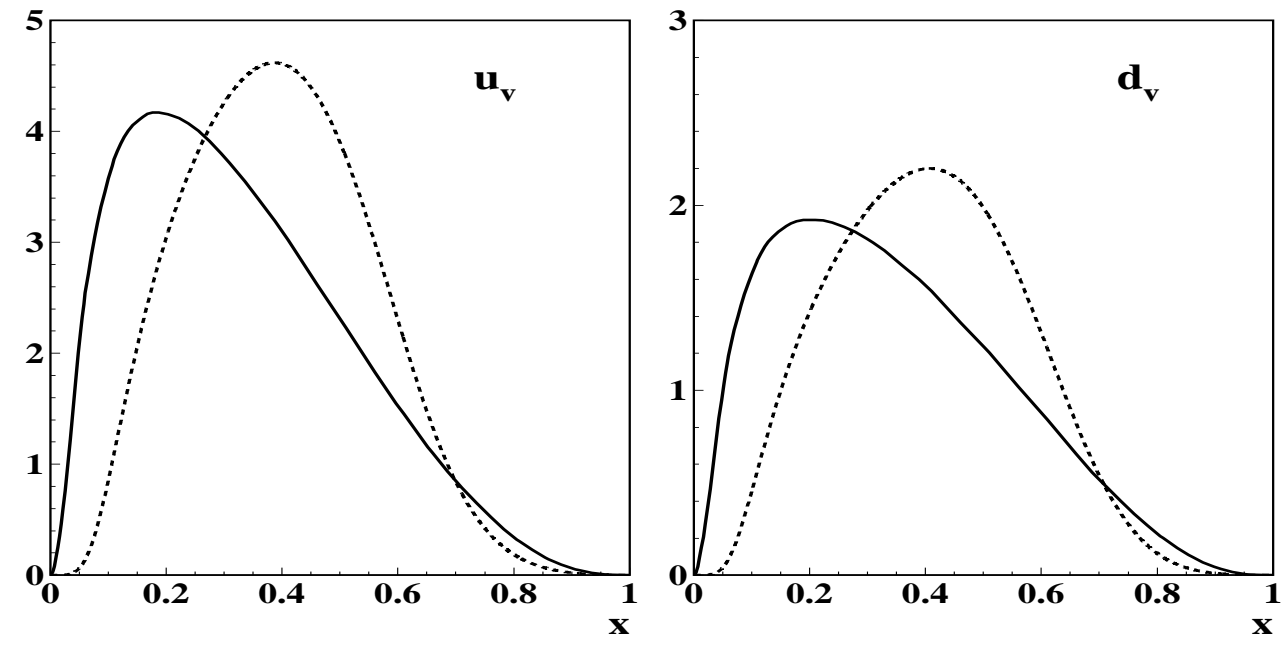

Figure 5: Parton distributions calculated in the GBE model for flavours $u$ (left panel) and $d$ (right panel) within the light-cone approach (solid curves) and the approach of Ref. [3]] with the inclusion of the support and flux factor corrections (dashed curves). 


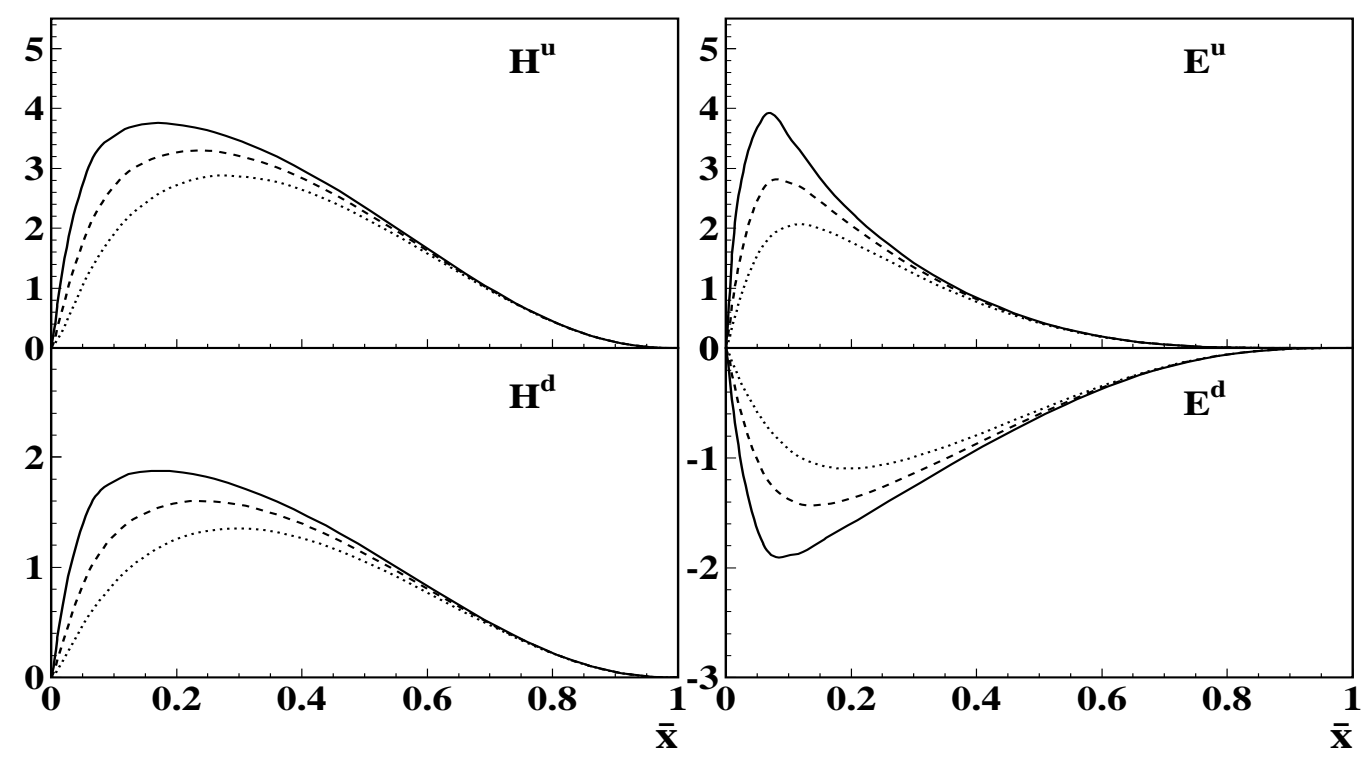

Figure 6: The same as in Fig. 2, but for the hypercentral model. 


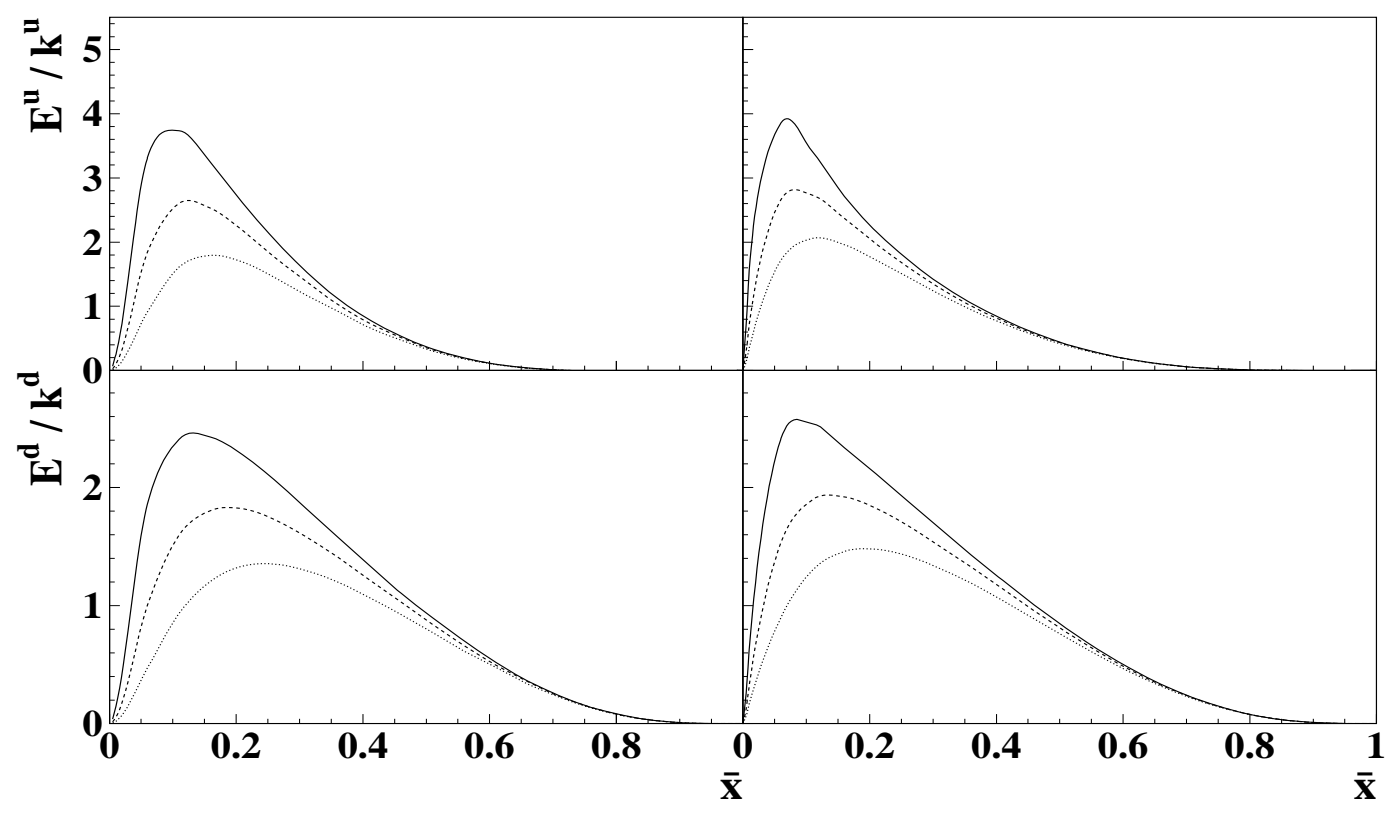

Figure 7: The ratio of the helicity-flip generalized parton distributions $E^{q}$ to the anomalous magnetic moment $k^{q}$ calculated in the GBE model (left panels) and in the hypercentral model (right panels) for flavours $u$ (upper panels) and $d$ (lower panels), at $\xi=0$ and different values of $t: t=0$ (solid curves), $t=-0.2(\mathrm{GeV})^{2}$ (dashed curves), $t=-0.5(\mathrm{GeV})^{2}$ (solid curves). The values of the quark anomalous magnetic moments predicted from the two models are: $k_{G B E}^{u}=1.33, k_{G B E}^{d}=-0.93, k_{h y p}^{u}=1.00$, $k_{\text {hyp }}^{u}=-0.74$. 


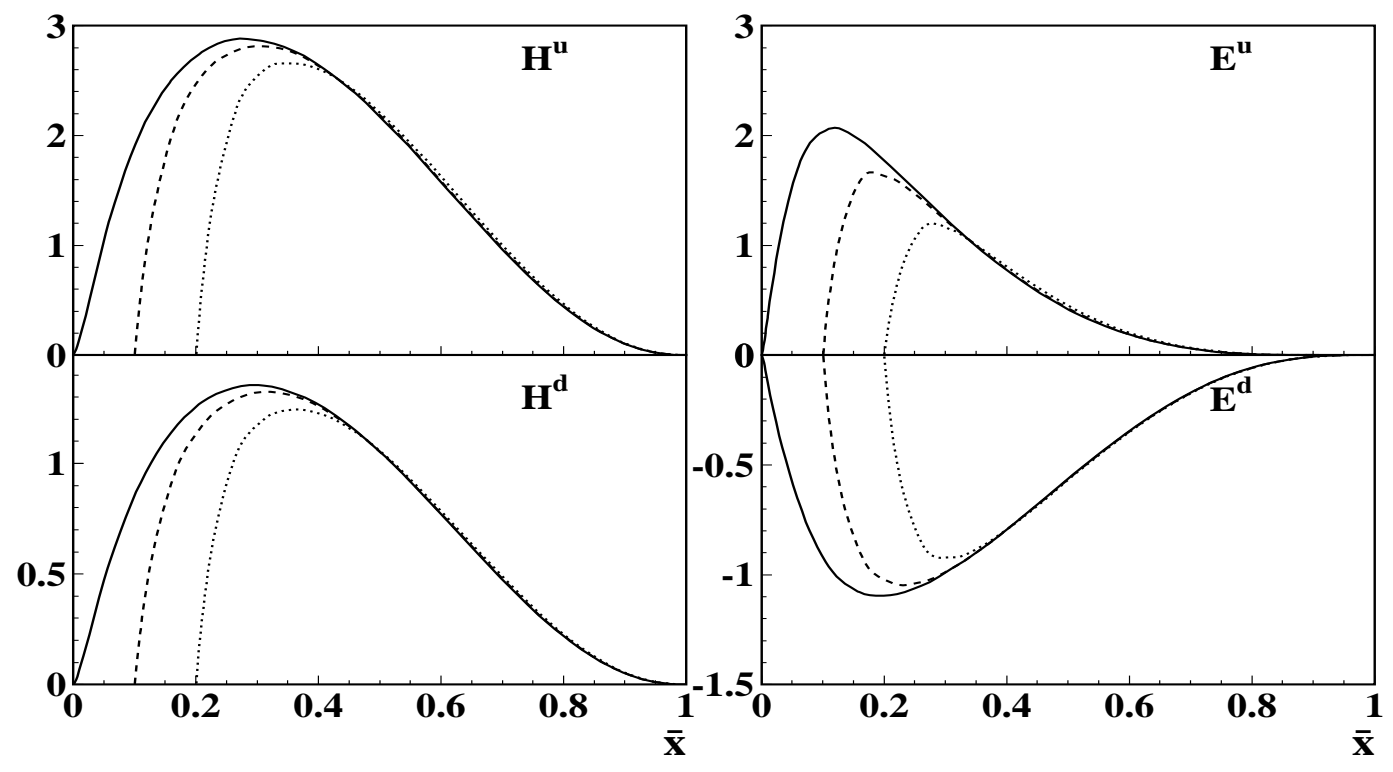

Figure 8: The same as in Fig. 4 , but for the hypercentral model. 


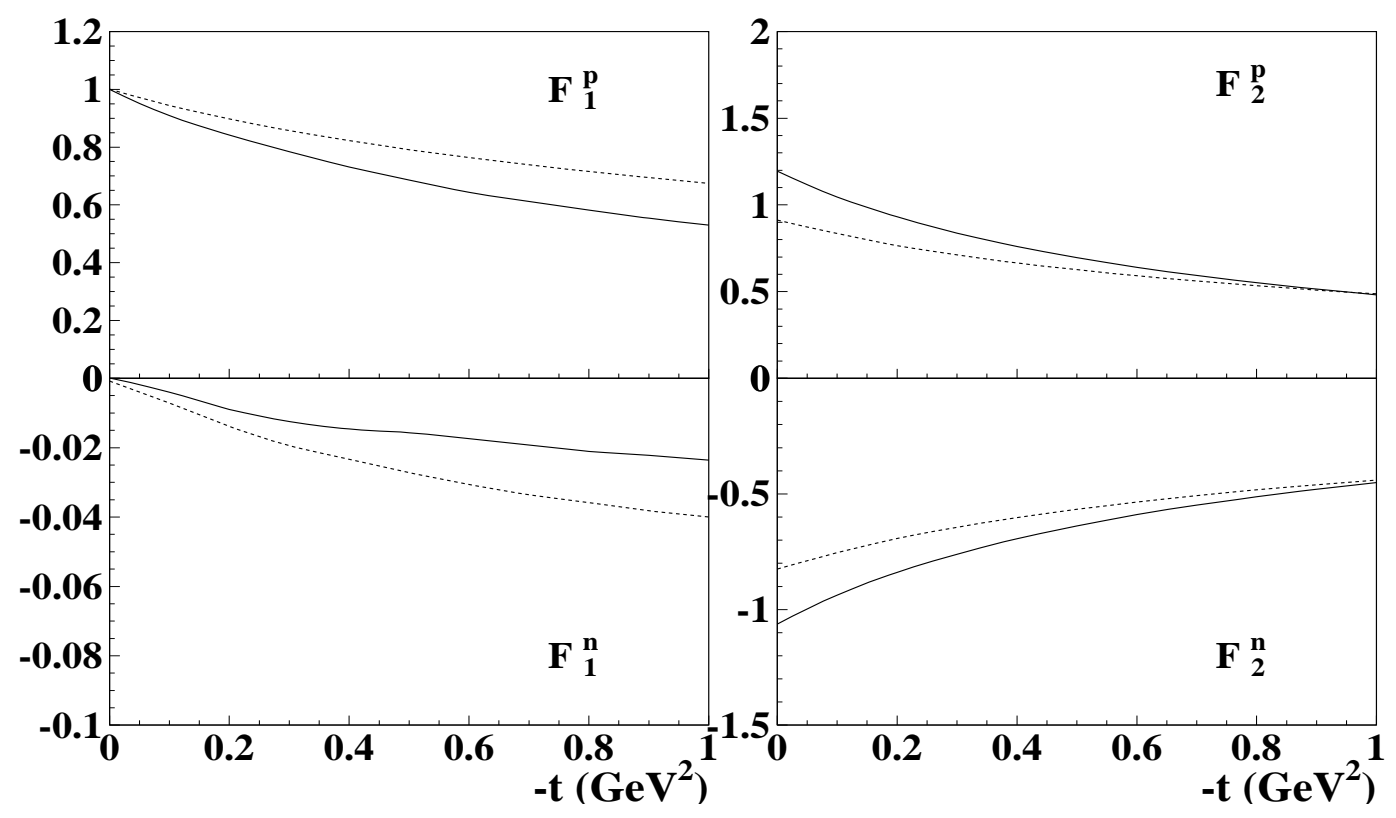

Figure 9: The Pauli (left panels) and Dirac (right panels) form factors calculated in the hypercentral model (dashed curves) and in the GBE model (solid curves) for the proton (upper panels) and the neutron (lower panels). 